\title{
Power Distributions of the APS Bending Magnets and Insertion Devices
}

\author{
Ali. M. Khounsary and Barry Lai \\ Advanced Photon Source \\ Argonne National Laboratory \\ Argonne, IL 60439
}

July 1992 


\section{Table of Contents}

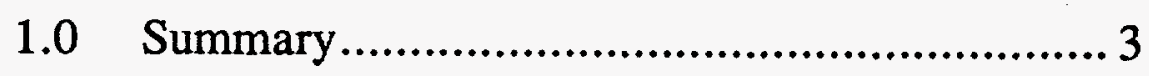

2.0 Introduction............................................ 3

3.0 Bending Magnet Power Distribution................. 4

\subsection{Analytical Curve Fits to the Bending} Magnet Power Profile .............................. 6

4.0 ID Power Distribution ................................. 6

\subsection{Analytical Curve Fit to the ID} Power Profile.

5.0 Concluding Remarks....................................9

6.0 Acknowledgment......................................10

7.0 References ............................................ 10

\section{DISCLAIMER}

This report was prepared as an account of work sponsored by an agency of the United States Government. Neither the United States Government nor any agency thereof, nor any of their employees, makes any warranty, express or implied, or assumes any legal liability or responsibility for the accuracy, completeness, or usefulness of any information, apparatus, product, or process disclosed, or represents that its use would not infringe privately owned rights. Reference herein to any specific commercial product, process, or service by trade name, trademark, manufacturer, or otherwise does not necessarily constitute or imply its endorsement, recommendation, or favoring by the United States Government or any agency thereof. The views and opinions of authors expressed herein do not necessarily state or reflect those of the United States Government or any agency thereof. 


\section{DISCLAIMER}

Portions of this document may be illegible in electronic image products. Images are produced from the best available original document. 


\subsection{Summary}

Relationships for computing the total power and the power density profiles of $\mathrm{x}$-ray beams generated by bending magnets (BMs) and insertion devices (IDs) are given. A FORTRAN program called POWER for computing the angular distribution of power generated by an ID is developed and outlined. This interactive program is available on the APS VAX system by typing the command POWER, and a graphic display of the power profile is provided within the program. For users requiring simple analytical formulas, curve fits are used to approximate the ID and BM power distributions. The accuracy of such analytical forms depends greatly on how the curve fits are made and is generally within a few percent.

\subsection{Introduction}

The spatial distributions of the power radiated by bending magnets and insertion devices are often required for calculating the thermal load on beamline and optical components that intercept the beams. When a synchrotron x-ray beam (with characteristic photon energy in the $\mathrm{keV}$ range) impinges upon highatomic-number materials, it is quite reasonable to assume that the incident beam power is deposited on the surface. As such, the incident beam power profile can be used directly in the thermal-structural computations necessary for the design of high heat load components.

For the case of higher photon energies and/or low-atomic-number materials (such as beryllium or carbon), the surface absorption model may no longer be valid. X-rays are absorbed and scattered throughout the depth of the material, and, depending on the thickness, a substantial amount of radiation may be transmitted through the material. In such cases, the in-depth absorption of the $\mathrm{x}$-ray beams must be computed. The PHOTON program [1] developed at NSLS is one of the numerical codes used for these computations. This program was written for bending magnet sources, and we have completed its extension to wiggler sources [2]. It will further be extended to undulator sources in the near future.

Here, the relationships for computing the power distribution from bending magnets and insertion devices are summarized and a computer program to obtain the power distribution of the insertion devices is outlined. Readers interested in the spectral distribution of the radiation from these sources are referred to references [3-5]. 


\subsection{Bending Magnet Power Distribution}

The radiation power generated from the passage of a charged particle beam through a dipole magnet is uniform in the plane of the orbiting particles. This plane is referred to as the horizontal plane. In the direction transverse to this plane, the bending magnet power has a nearly Gaussian profile with its peak in the horizontal plane.

Denoting the vertical angle measured from the horizontal plane by $\psi$ and the angle in the horizontal plane by $\theta$ (Fig. 1), the bending magnet power distribution is given by [3]:

$$
\frac{d^{2} \mathrm{P}}{\mathrm{d} \theta \mathrm{d} \psi}=\frac{1.44 \times 10^{-18}}{\rho[\mathrm{m}]} \gamma^{5} \mathrm{~F}(\gamma \psi) \quad\left[\mathrm{W} / \mathrm{mrad}^{2}-\mathrm{mA}\right]
$$

where $\mathrm{d}^{2} \mathrm{P} / \mathrm{d} \theta \mathrm{d} \psi \quad$ [in $\left.\mathrm{W} / \mathrm{mrad} \theta-\mathrm{mrad} \psi-\mathrm{mA}\right]$ is the radiated power from a bending magnet of radius $\rho[\mathrm{m}]$ at a vertical angle $\psi$ into a one $\operatorname{mrad}^{2}$ "solid" angle with a charged particle beam current of one $\mathrm{mA}$. Although a bending magnet creates a fan of radiation horizontally, only part of that radiation is accepted by the front-end aperture and the beamline, which then restrict the acceptance in $\theta$. The factor $F(\gamma \psi)$ is given by:

$$
F(\gamma \psi)=\left(1+\gamma^{2} \psi^{2}\right)^{-5 / 2}\left[\frac{7}{16}+\frac{5}{16} \frac{\gamma^{2} \psi^{2}}{\left(1+\gamma^{2} \psi^{2}\right)}\right]
$$

with $\gamma$ being the relativistic mass factor given by:

$$
\gamma=1957 \mathrm{E}_{\mathrm{r}}[\mathrm{GeV}]
$$

where $E_{r}[\mathrm{GeV}]$ is the storage ring energy, and $1 / \gamma$ is in radians.

By integrating Eqn. (1) with respect to $\psi$, and noting that

$$
\int_{-\infty}^{\infty} F(\gamma \psi) d(\gamma \psi)=\frac{2}{3}
$$

one gets 


$$
\frac{\mathrm{dP}}{\mathrm{d} \theta}=0.96 \times 10^{-15} \frac{\gamma^{4}}{\rho[\mathrm{m}]} \quad[\mathrm{W} / \mathrm{mrad} \theta-\mathrm{mA}]
$$

where $\mathrm{dP} / \mathrm{d} \theta$ is the vertically integrated bending magnet power in $\mathrm{W} / \mathrm{mrad} \theta-\mathrm{mA}$.

The peak power density from Eqn. (1) is

$$
\frac{d^{2} \mathrm{P}}{\mathrm{d} \theta \mathrm{d} \psi} I_{\psi=0}=\frac{7}{16} \times \frac{1.44 \times 10^{-18}}{\rho} \gamma^{5}=0.63 \times 10^{-18} \frac{\gamma^{5}}{\rho[\mathrm{m}]} \quad\left[\mathrm{W} / \mathrm{mrad}^{2}-\mathrm{mA}\right]
$$

For the APS storage ring,

$$
\begin{aligned}
& 1 / \gamma=1 /\left(1957 \mathrm{E}_{\mathrm{r}}[\mathrm{GeV}]\right)=73 \mu \mathrm{rad}, \text { and } \\
& \rho=38.96 \mathrm{~m} .
\end{aligned}
$$

There are 80 bending magnets on the storage ring, each covering an arc of $4.5^{\circ}$ or $78.5 \mathrm{mrad}$. Each sector of the ring consists of two bending magnets and one ID so that there are two bending magnets between two adjacent IDs. Radiation from the bending magnet downstream of an ID is totally absorbed while only 6 mrad of the radiation from the bending magnet upstream is extracted. Using Eqns. (4) and (5), the radiated power and peak power density of the extracted beam are, respectively, for a $100 \mathrm{~mA}$ of beam current:

$$
\mathrm{P}=0.96 \times 10^{-15} \frac{\gamma^{4}}{\rho[\mathrm{m}]} \times 100[\mathrm{~mA}] \times 6[\mathrm{mrad}]=520.8 \mathrm{~W}
$$

and

$$
\frac{d^{2} \mathrm{P}}{\mathrm{d} \theta \mathrm{d} \psi} I_{\psi=0}=0.63 \times 10^{-18} \frac{\gamma^{5}}{\rho[\mathrm{m}]} \times 100[\mathrm{~mA}]=780 \mathrm{~W} / \mathrm{mrad}^{2}
$$

The total power generated by each bending magnet into the full $78.5 \mathrm{mrad}$ horizontally is $6.8 \mathrm{~kW}$. 


\subsection{Analytical Curve Fits to the Bending Magnet Power Profile}

The $\mathrm{BM}$ power density profile in the vertical direction is given by the function $F(\gamma \psi)$ of Eqn. 2a, and this is shown in Fig. 2. Because of its resemblance to the more easily-handled Gaussian distribution, the following function is used in curve-fitting [5]:

$$
F_{a}(\gamma \psi)=\frac{7}{16} e^{\frac{-(\gamma \psi)^{2}}{2 \sigma^{2}}}
$$

with $\sigma=0.608$. This standard deviation $\sigma$ ensure conservation of the total power, while the peak power density is conserved by the factor of $7 / 16$. Also note from Fig. 2 that the FWHM of the theoretical power profile (Eqn. 2a) is 1.30 , and the FWHM of the Gaussian fit (Eqn. 6) is 1.43. For calculations more sensitive to the peak power density, a smaller $\sigma$ may instead be used in Eqn. 6 .

\subsection{ID Power Distribution}

The power distribution for an ID source varies both vertically and horizontally. Using the earlier notations, the power density from an ID (based on a sinusoidal positron trajectory) can be written as [6]

$$
\frac{d^{2} P}{d \theta d \psi}=P_{T} \frac{21 \gamma^{2}}{16 \pi K} G(K) f_{K}(\gamma \theta, \gamma \psi)
$$

where $\mathrm{P}_{\mathrm{T}}$ is the total $\mathrm{ID}$ power. $\mathrm{K}$ is the deflection parameter given by

$$
\mathrm{K}=0.934 \lambda_{\mathrm{u}}[\mathrm{cm}] \mathrm{B}_{0}[\mathrm{~T}]
$$

and $\lambda_{u}$ is the ID period, and $B_{0}$ is the peak magnetic field. $G(K)$ is a normalization factor given by

$$
G(K)=K \frac{\left(K^{6}+\frac{24}{7} K^{4}+4 K^{2}+\frac{16}{7}\right)}{\left(1+K^{2}\right)^{7 / 2}}
$$


The angular dependence $f_{K}(\gamma \theta, \gamma \psi)$ of the radiation from an ID is given by

$$
f_{K}(\gamma \theta, \gamma \psi)=\frac{16 K}{7 \pi G(K)} \int_{-\pi}^{\pi} d \alpha\left(\frac{1}{D^{3}}-\frac{4(\gamma \theta-K \cos \alpha)^{2}}{D^{5}}\right) \sin ^{2} \alpha
$$

where

$$
D=1+(\gamma \psi)^{2}+(\gamma \theta-K \cos \alpha)^{2},
$$

and $f_{K}(\gamma \theta, \gamma \psi)$ is normalized such that $f_{K}(0,0)=1.0$.

The total power $\mathrm{P}_{\mathrm{T}}$ in Eqn. (7) is given by .

$$
\mathrm{P}_{\mathrm{T}}[\mathrm{kW}]=0.633 \mathrm{E}_{\mathrm{r}}^{2}[\mathrm{GeV}] \mathrm{B}_{0}^{2}[\mathrm{~T}] \mathrm{L}[\mathrm{m}] \mathrm{I}[\mathrm{A}]
$$

where $\mathrm{L}$ is the device length and $\mathrm{I}$ is the particle beam current.

From Eqn. (7), the peak power density for an ID is

$$
\left.\frac{\mathrm{d}^{2} \mathrm{P}}{\mathrm{d} \theta \mathrm{d} \psi}\right|_{(0,0)}=10.85 \mathrm{~B}_{0}[\mathrm{~T}] \mathrm{E}_{\mathrm{r}}^{4}[\mathrm{GeV}] \mathrm{I}[\mathrm{A}] \mathrm{N} \mathrm{G}(\mathrm{K}) \quad\left[\mathrm{W} / \mathrm{mrad}^{2}\right]
$$

where $\mathrm{N}$ is the number of undulator periods. Note that

$$
\mathrm{L}=\mathrm{N} \lambda_{\mathrm{u}} \text {. }
$$

Obtaining ID power involves integrating Eqn. (10) to compute $f_{K}(\gamma \theta, \gamma \psi)$, and a further integration of $f_{K}$ over $\gamma \theta$ and $\gamma \psi$ to obtain the total power. An interactive program named POWER is written and described here for these integrations.

To run this program on the APS VAX cluster, enter the command POWER. Fig. 3 shows an example for the case of APS Undulator A. Default parameters for Undulator A have been stored in the file UA.DEF; similarly, the parameters for Wiggler A are stored in WA.DEF, and those for Wiggler B in WB.DEF. These parameter files are available in the directory 
APSD19:[AMK.PUBLIC], and the user should copy the one that he needs to his own directory before running POWER. From your directory on VAX, type:

\section{COPY APSD19:[AMK.PUBLIC]filename.ext [ ]}

to copy the desired parameter file to your directory. When running the program, you will be asked for the name of the parameter file. You also will be able to modify any of the parameters from the parameter file interactively. The new set of parameters will then be written to the user's directory as a new version of the same parameter file name.

The power density calculation is performed for the horizontal angle $\theta$ from 0 up to $\gamma \theta_{\max }$ [gamma_theta_max] in [m_theta] number of steps, and the results will be printed out at every [mp] step. In the vertical direction, the variables [gamma_psi_max], [n_psi], and [np] are defined similarly. The power distribution in the vertical direction is contained within an angle of $\psi \sim 1 / \gamma$, and so [gamma_psi_max] equal to 2-3 is appropriate for most cases. On the other hand, the horizontal power distribution depends on $K$, and so a [gamma_theta_max] value equal to or larger than $\mathrm{K}$ should be used. To compute the power density at a particular $(\gamma \theta, \gamma \psi)$ within the angular limits specified, the integration over $\alpha$ in Eqn. 10 is performed by the program. This is accomplished by a Gaussian quadrature with [ng] points. If [ng] is too small, then the condition that $f_{K}(0,0)=1.0$ will not be satisfied, and the program will ask the user to increase the number of integration points [ng]. The number of points [ng] must be odd, and typically a minimum of 201 points should be used. At the present time, the number of angular points [m_theta] and [n_psi] and the number of integration points [ng] are all limited to a maximum of 501 .

A sample output of the program is shown in Fig. 4. For every printed angle, $\mathrm{d}^{2} \mathrm{P} / \mathrm{d} \theta \mathrm{d} \psi$ (in $\mathrm{kW} / \mathrm{mrad}^{2}$ ) is listed in the last column. At the end of the output, the total power calculated from numerically integrating $\mathrm{d}^{2} \mathrm{P} / \mathrm{d} \theta \mathrm{d} \psi$ over the angular range is compared with the total power predicted from Eq. 12. Typically, agreement within a few percent is obtained. The power distribution can also be graphically displayed from inside the program using DISSPLA. Although the calculation is done in one quadrant for symmetry reasons, the power distribution can be displayed over 1,2 , or 4 quadrants. The graphic output can also be stored in a PostScript file for obtaining a hardcopy. Fig. 5 shows the results for Undulator A (in the case of $1.15 \mathrm{~cm}$ gap, $\mathrm{K}=2.23$ ), using the default parameter files UA.DEF as the input. 


\subsection{Analytical Curve Fit to the ID Power Profile}

For some thermal/structural calculations it is much more convenient to use simple analytical forms to describe the power density distribution instead of the exact results from POWER. This is indeed necessary if an analytical solution is sought. A suggestion for approximating the power density for an ID is made here. It is seen from Fig. 5 that the ID power density profile is approximately Gaussian in the vertical direction and parabolic in the horizontal direction. Thus, it may be possible to approximate the power density profile by using the product of such functions. An appropriate functional form can be obtained by fitting a Gaussian curve $\mathrm{P}_{\mathrm{a}}(0, \psi)$ to the vertical power profile and a parabolic curve $\mathrm{P}_{\mathrm{a}}(\theta, 0)$ to the horizontal power profile. These are shown in Fig. 6 for the APS Undulator $A$ in the close gap case. Note that the curve fits are forced to match the peak power density at $(\theta=0, \psi=0)$.

The analytic approximation $\mathrm{P}_{\mathrm{a}}$ to the power density is given by:

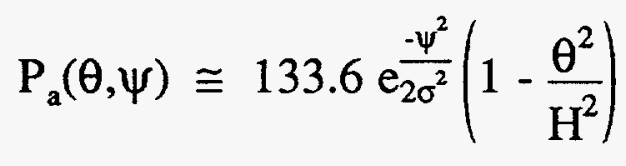

where $\sigma=0.0417 \mathrm{mrad}$ is the Gaussian standard deviation, and $\mathrm{H}=0.2 \mathrm{mrad}$ is the horizontal extent of the power density profile. If Eqn. 15 is integrated over $\psi$ in $(-\infty$ to $\infty)$ and $\theta$ in (-H to $H)$, one obtains for the total power

$$
\mathrm{P}_{\mathrm{T}}=133.6(\sigma \sqrt{2 \pi})\left(\frac{4 \mathrm{H}}{3}\right)=3.7 \mathrm{~kW} \text {, }
$$

which is about $3 \%$ less than the theoretical value of $3.8 \mathrm{~kW}$. Note that, with a slightly larger value of $\mathrm{H}$, it is possible to preserve the total power as well.

The curve-fitting procedure outlined here can be used to obtain an approximate analytical relationship for any ID power profile. One should note, however, that the present Gaussian-parabolic form may not be appropriate for small values of $\mathrm{K}$. The usefulness of Eqn. 15 lies in the fact that it is valid for Undulator A at close gap (worst heating condition) where most thermal analyses are carried out.

\subsection{Concluding Remarks}

In all the power distribution discussions here, ideal magnetic devices and zero-emittance particle beams are assumed. For the APS IDs, the angular divergences in the vertical and horizontal directions are 9 and $24 \mu \mathrm{rad}$ 
respectively [4], which are small compared to the $73 \mu$ rad natural opening of the beam from the $7-\mathrm{GeV}$ storage ring. The emittance only broadens the power profile slightly, and thus the zero-emittance assumption is a conservative one from a thermal point of view. However, the emittance should be taken into account in the case of an undulator with small values of $\mathrm{K}$. For example, when $\mathrm{K}=0.3$, the zero-emittance horizontal power profile has a standard deviation of 23 $\mu \mathrm{rad}$ for Undulator $\mathrm{A}$. This is then comparable to the $24 \mu \mathrm{rad}$ horizontal divergence of the particle beam, and therefore, the beam power profile in that direction is considerably wider than that obtained from the zero-emittance relationships outlined in this note.

\subsection{Acknowledgment}

The authors wish to thank R. D. Dejus, G.K. Shenoy, and P. J. Viccaro for reviewing this note and for their helpful comments.

\subsection{References}

1. D. Chapman, N. Gmuer, N. Lazarz, and W. Thomlinson, Nuc. Instr. and Meth. A266 (1988) 191.

2. A. M. Khounsary, R. J. Dejus, D. A. Brown, P. J. Viccaro, to be submitted for publication as an LS Note, Advanced Photon Source, Argonne National Laboratory, 1992.

3. G. K. Green, "Spectra and Optics of Synchrotron Radiation," in Proposal for a National Synchrotron Light Source, Brookhaven National Laboratory, BNL50595 (1977).

4. G. K. Shenoy, P. J. Viccaro, D. M. Mills, "Characteristics of the 7-GeV Advanced Photon Source: A Guide for Users," Argonne National Laboratory Report, ANL-88-9 (1988).

5. S. Krinsky, M. L. Perlman, R. E. Watson, Handbook on Synchrotron Radiation Vol. la, ed. E. E. Koch (North-Holland, Amsterdam, 1983).

6. K. J. Kim, "Angular Distribution of Undulator Power for an Arbitrary Deflection Parameter K," Nuc. Instr. and Meth. A246, pp. 67-70 (1986).

7. A.M. Khounsary, P. J. Viccaro, and T.M. Kuzay, "Filter and Window Assemblies for High Power Insertion Device Synchrotron Radiation Sources," SPIE Vol. 1345, pp.42-54, (1990). 


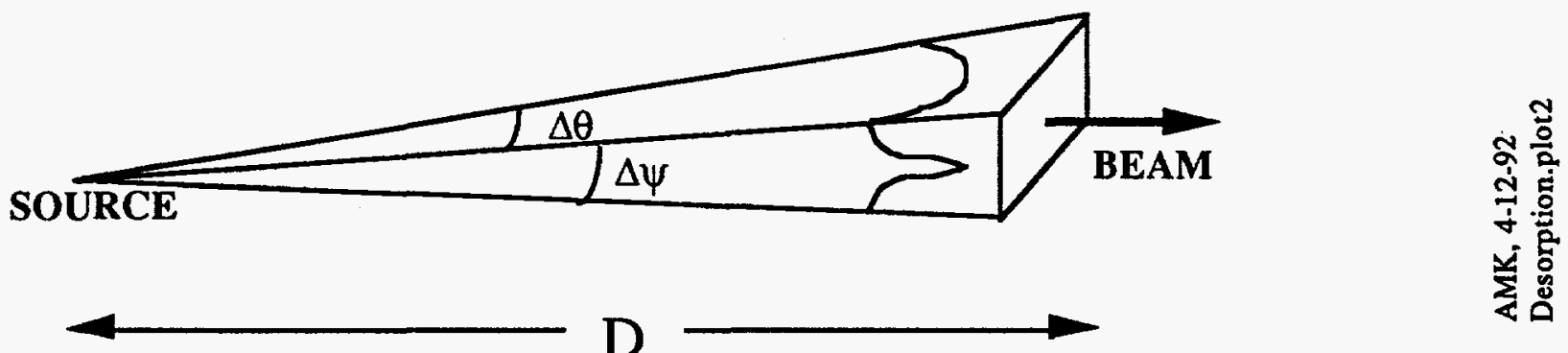

Figure 1: Incident beam on a surface located at a distance $D$ from the source. $\Delta \theta$ and $\Delta \psi$ are the horizontal and vertical extents of the beam. Also noted are the horizontal and vertical profiles of a typical undulator power envelope.

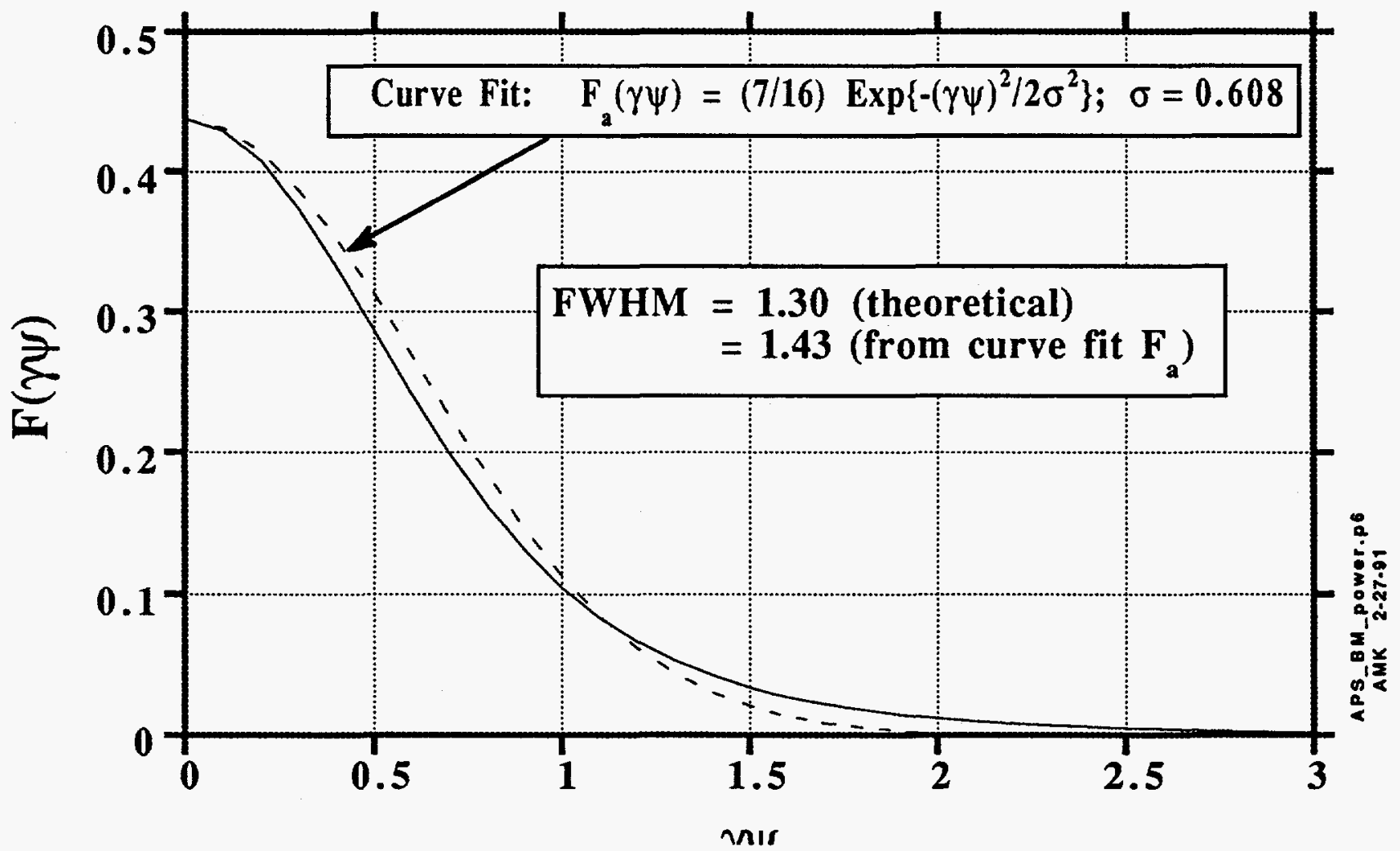

Figure 2: Gaussian curve fit to the vertical power profile of a bending magnet source such that both the peak and total power are conserved. 
Fig. 3 Example Run of the POWER Program

\author{
ANLAPS $>$ COPY APSD19:[AMK.PUBLIC]UA.DEF [] \\ ANLAPS $>$ POWER \\ Name of file with ID parameters $[<C R>$ if none] ? \\ UA.DEF
}

Insertion Device Power Program, April 1992

$\begin{aligned} \text { I N P U T D A T A } & \\ \text { I U********************* } & \\ \text { 1. Beam Energy (GeV) } & =7.0000 \\ \text { 2. Beam Current (A) } & =0.1000 \\ \text { 3. Period Length (cm) } & =3.3000 \\ \text { 4. K } & =2.2300 \\ \text { 5. gamma_theta_max } & =4.0000 \\ \text { 6. gamma_psi_max } & =2.5000 \\ \text { 7. Number of period } & =72 \\ \text { 8. m_theta (theta divisions) } & =25 \\ \text { 9. n_psi (psi divisions) } & =20 \\ \text { 10. mp, theta print frequency } & =1 \\ \text { 11. np, psi print frequency } & =1 \\ \text { 12. ng, integration points [odd] } & =201\end{aligned}$

Are these correct? $[1] / \mathrm{Y},[0] / \mathrm{N}$

0

enter index followed by correct value:

12,251

I N P U T DATA

$* * * * * * * * * * * * * * * * * * *$

1. Beam Energy $(\mathrm{GeV}) \quad=7.0000$

2. Beam Current $(\mathrm{A}) \quad=0.1000$

3. Period Length $(\mathrm{cm}) \quad=3.3000$

4. $\mathrm{K}=2.2300$

5. gamma_theta_max $\quad=4.0000$

6. gamma_psi_max $=2.5000$ 
7. Number of period $=72$

8. $\mathrm{m}$-theta (theta divisions) $=25$

9. n_psi (psi divisions) $=20$

10. $\mathrm{mp}$, theta print frequency $=1$

11. np, psi print frequency $=1$

12. $\mathrm{ng}$, integration points [odd] $=251$

Are these correct? [1]/Y, [0]/N

1

$* * * * * * * * * * * * * * * * * * * * * * * * * * * * * * * * * * * * * * * * * * * * * * * * * * * *$

Power distribution was calculated and stored in FOR016.dat

Graphic options for power distribution. Enter:

[0] for none

[1] for displaying on the screen

[2] for storing in PostScript file

[3] for both

1

Do you want to display the power distribution in:

[1] one quadrant

[2] two quadrants (full gamma-psi)

[3] two quadrants (full gamma-theta)

[4] all four quadrants

1

For graphics output on your screen, please specify:

Enter Output Device type(press ENTER to list choices):

TEKTRONIX

Enter model:

4010C

Another plot ? 1[y],0[n]

0

Another run ? 1[y],0[n]

o

FORTRAN STOP 
Fig. 4 Power Density Output File (FOR016.DAT)

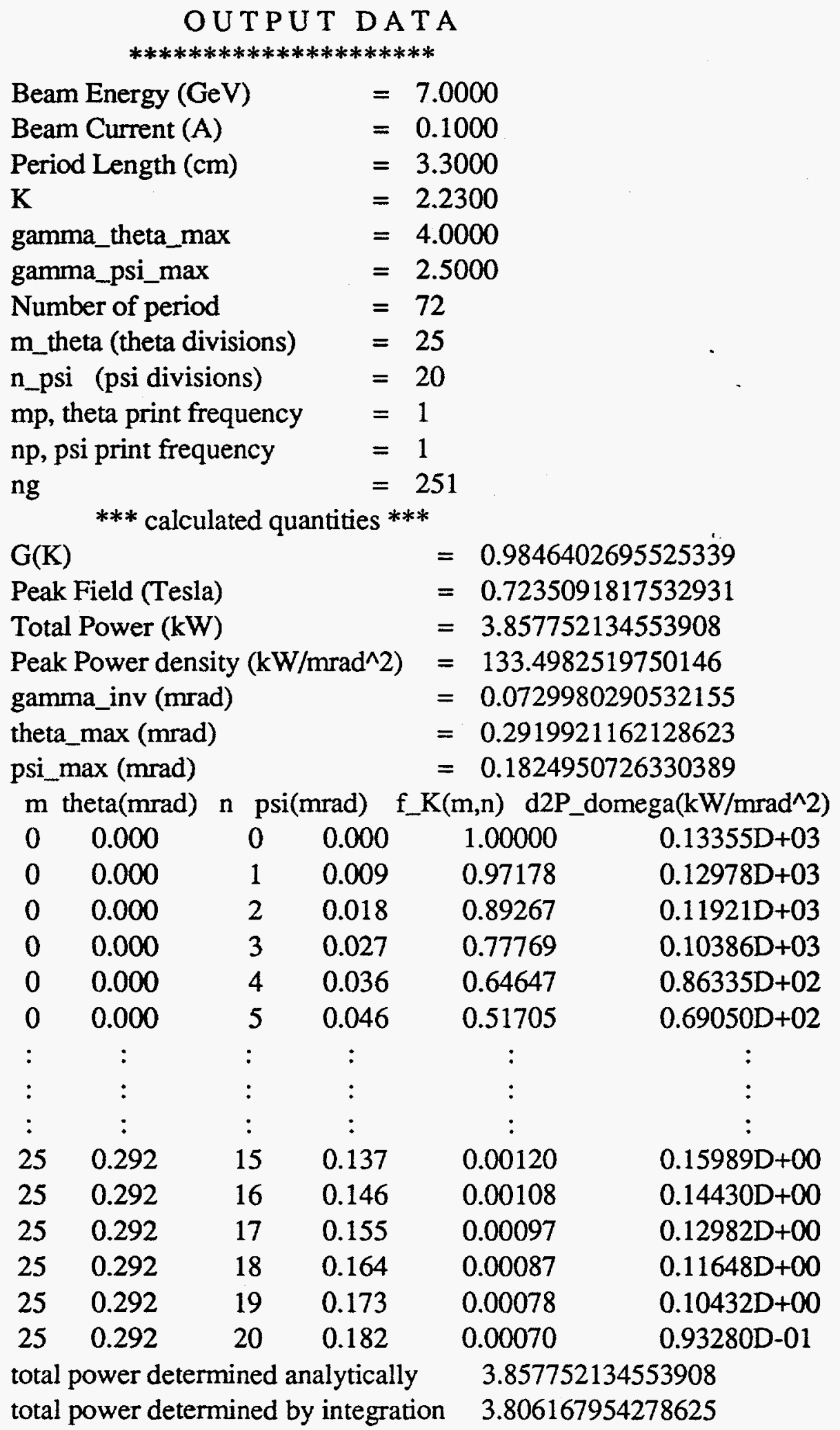




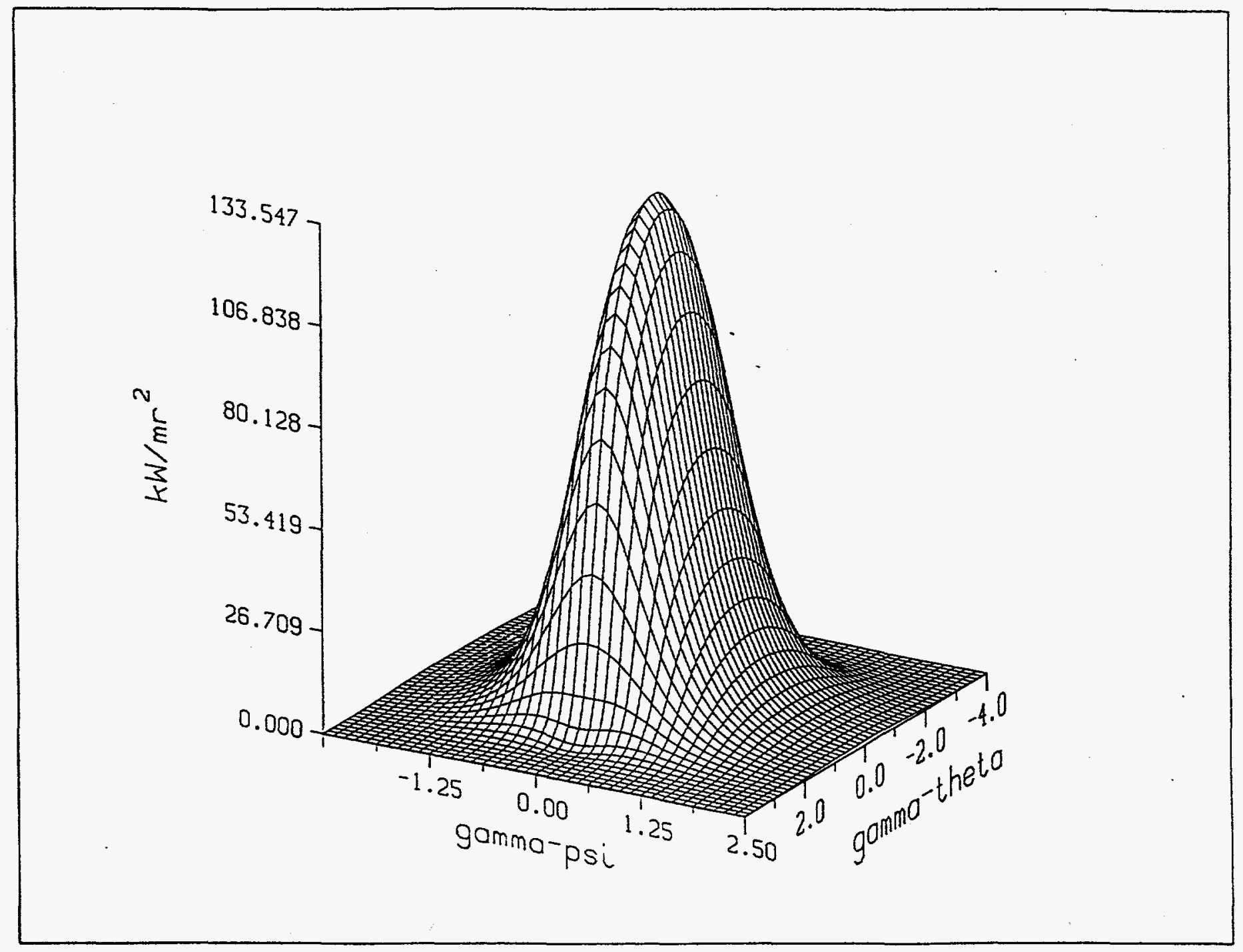

Fig. 5 Power density profile of APS Undulator A as computed by POWER (K = 2.23). 

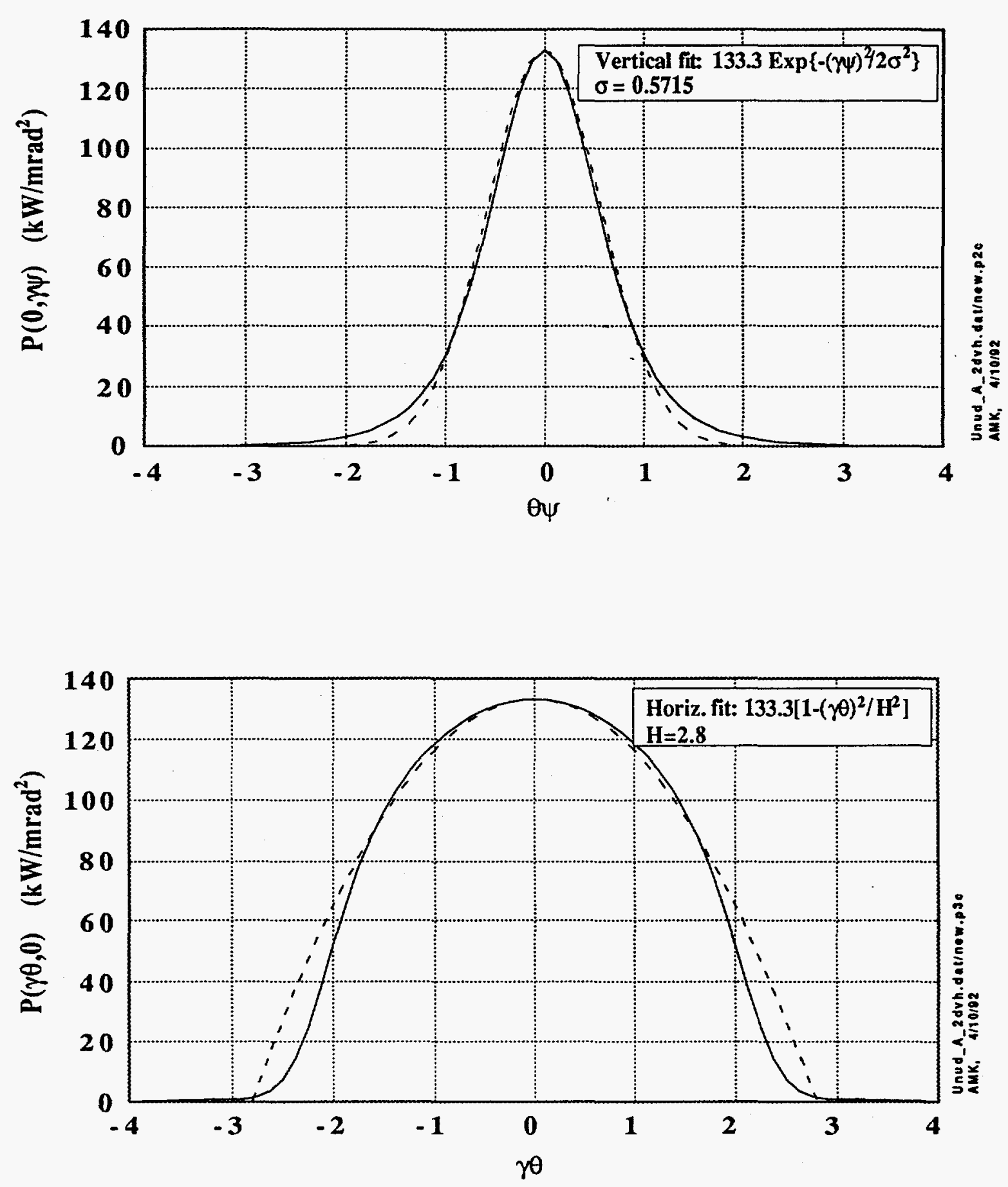

Figure 6: Power density profiles of the APS Undulator A in the vertical direction at $\theta=0$ (top) and in the horizontal direction at $\psi=0$ (bottom) are shown in solid lines. Curve fits to these profiles are also shown graphically and analytically. 